\title{
Experimental Evolution under Fluctuating Thermal Conditions Does Not Reproduce Patterns of Adaptive Clinal Differentiation in Drosophila melanogaster
}

\author{
Vanessa Kellermann, ${ }^{1,2, \star}$ Ary A. Hoffmann, ${ }^{3}$ Torsten Nygaard Kristensen, ${ }^{4}$ \\ Neda Nasiri Moghadam, ${ }^{1}$ and Volker Loeschcke ${ }^{1}$
}

\begin{abstract}
1. Department of Bioscience, Aarhus University, Ny Munkegade 114-116, DK-8000 Aarhus C, Denmark; 2. Biological Sciences, Monash University, Clayton 3800, Victoria, Australia; 3. Departments of Zoology and Genetics, Bio21 Institute, University of Melbourne, Parkville 3010, Victoria, Australia; 4. Department of Chemistry and Bioscience, Aalborg University, Fredrik Bajers Vej 7H, DK-9220 Aalborg East, Denmark
\end{abstract}

Submitted November 13, 2014; Accepted June 22, 2015; Electronically published September 18, 2015

Online enhancement: appendix. Dryad data: http://dx.doi.org/10.5061/dryad.t16c5.

\begin{abstract}
Aвstract: Experimental evolution can be a useful tool for testing the impact of environmental factors on adaptive changes in populations, and this approach is being increasingly used to understand the potential for evolutionary responses in populations under changing climates. However, selective factors will often be more complex in natural populations than in laboratory environments and produce different patterns of adaptive differentiation. Here we test the ability of laboratory experimental evolution under different temperature cycles to reproduce well-known patterns of clinal variation in Drosophila melanogaster. Six fluctuating thermal regimes mimicking the natural temperature conditions along the east coast of Australia were initiated. Contrary to expectations, on the basis of field patterns there was no evidence for adaptation to thermal regimes as reflected by changes in cold and heat resistance after 1-3 years of laboratory natural selection. While laboratory evolution led to changes in starvation resistance, development time, and body size, patterns were not consistent with those seen in natural populations. These findings highlight the complexity of factors affecting trait evolution in natural populations and indicate that caution is required when inferring likely evolutionary responses from the outcome of experimental evolution studies.
\end{abstract}

Keywords: latitudinal gradient, fluctuating temperature, experimental evolution, thermal resistance, laboratory natural selection.

\section{Introduction}

The ability of species to adapt to global change in situ will often depend on the underlying genetic variation for key traits and/or the ability to buffer temperature increases through plastic responses (Chevin et al. 2010; Hoffmann

\footnotetext{
* Corresponding author; e-mail: vanessa.kellermann@monash.edu.
}

Am. Nat. 2015. Vol. 186, pp. 582-593. (C) 2015 by The University of Chicago. 0003-0147/2015/18605-55891\$15.00. All rights reserved. DOI: $10.1086 / 683252$ and Sgro 2011). There is a growing interest in assessing this potential across a range of life forms (Merila and Hendry 2014). At present, the available data are limited (Hoffmann and Sgro 2011; Merila and Hendry 2014), but some information is emerging from a range of approaches involving longitudinal studies (common garden, animal model, and/or molecular changes), space-for-time experiments (inferring temporal relationships from spatial studies via commongarden and transplant experiments), and experimental evolution (field and laboratory selection). Nevertheless, these approaches have their challenges. Longitudinal studies depend on the availability of past data and the ease with which data sets are comparable across time (i.e., methodological differences). Space-for-time experiments may not reliably predict future responses and may be dependent on the speed of environmental and evolutionary change. Experimental evolution studies can be a powerful proof-of-principle tool, but results might not be the same as evolutionary responses in natural populations, as the environment in the laboratory may be too simple.

Experimental evolution is nevertheless seen as a particularly attractive approach for assessing adaptive potential because it provides an indication of the speed of an evolutionary response to environmental variables that can be controlled. Studies can be undertaken under laboratory conditions (Collins and Bell 2004) or performed in the field via microcosm experiments (Hargreaves et al. 2014). Laboratory studies may involve artificial selection, where phenotypic values - and thus fitness - are predetermined by the experimenter, or laboratory "natural" selection, where no particular trait is selected on but rather the environment is manipulated and evolution is monitored in key traits (for a detailed review, see Scheiner 2002; Kawecki et al. 2012). With artificial selection, it can be difficult to 
represent traits under selection and capture complex interactions, whereas laboratory natural selection circumvents these issues by focussing on the organismal level. Such methods have been used increasingly as a tool to examine the potential for evolutionary responses in species with respect to climate change (Collins and Bell 2004; Collins 2011; Lohbeck et al. 2012; Schou et al. 2014).

Although experimental evolution can help identify the importance of particular factors underlying trait evolution, there are only a handful of attempts to link the results from such studies to longitudinal or geographic patterns in natural populations (Cavicchi et al. 1985; Robinson et al. 2000; Santos et al. 2005; Barrett et al. 2011). In natural environments, trait evolution is potentially driven by a complex set of interactions involving the abiotic and biotic environment. Predictions derived from the relatively simple environments used in experimental evolution studies may not be meaningful in natural populations. It is therefore of interest to compare evolutionary responses in natural populations to those attained through laboratory experimental evolution.

The latitudinal trait clines present in Drosophila species provide an opportunity to link experimental evolution to patterns seen in space, particularly those patterns driven by temperature, which is thought to be a key driver of clinal trait variation given its relationship with latitude (Parkash and Munjal 2000; Hoffmann et al. 2003). One of the most well-studied latitudinal trait clines is that of Drosophila melanogaster along the east coast of Australia (Hoffmann and Weeks 2007). Here, opposing clines have been detected for cold and heat resistance as well as for body size (Hoffmann et al. 2002; van Heerwaarden and Sgro 2011). These studies have shown that cold resistance and body size (James et al. 1995; Hoffmann et al. 2002) consistently increase with latitude, while heat resistance decreases with latitude (Hoffmann et al. 2002). There has been controversy around the measurement of heat resistance in natural populations (Rezende et al. 2011; Overgaard et al. 2012), but the latitudinal clinal pattern for this trait exists regardless of how resistance is measured (Sgro et al. 2010).

Here we examine whether laboratory experimental evolution studies involving different temperature regimes can be linked to these clinal patterns in D. melanogaster. We initiated 6 fluctuating thermal regimes contrasting the warmer Australian northern sites with average temperatures at approximately $26.9^{\circ} \mathrm{C}$ (lower latitudes) to the cooler temperatures of southern Australia (higher latitudes) with average summer and winter temperatures of $16.7^{\circ}$ and $13.4^{\circ} \mathrm{C}$, respectively. For each temperature, we initiated replicate "lines" from one large population collected centrally from the cline. To compare results with those from previous experimental evolution studies, three constant temperatures were also included in the design. After 1-3 years of labora- tory evolution, traits linked to tolerance (heat knockdown, chill coma recovery, desiccation, and starvation resistance) as well as life-history traits (development time, egg-to-adult viability, fecundity, and body size) were assessed. If temperature conditions alone were responsible for clinal patterns and if experimental evolution reflected processes in the wild, we anticipated that changes in the simulated laboratory temperature clines would match those observed in the field. Instead, a much more complicated picture emerged.

\section{Methods}

Flies were collected during the period March-April 2009 from Coffs Harbour, Australia (lat. $30.37^{\circ} \mathrm{S}$ ) via sweep netting over rotten fruits. Females from these collections were initiated as isofemale lines, and each line was maintained at a substantial size (around 100 individuals) to reduce inbreeding and maintained on an oat-yeast sugar medium (Leeds) at $25^{\circ} \mathrm{C}$ under a $12: 12$-h light-dark cycle. After 5 generations of laboratory culture, 180 isofemale lines were pooled to generate one large mass-bred population by sampling 10 females and 10 males from each line. We initiated the mass-bred population with high numbers of isofemale lines to both maximize genetic variation and limit linkage disequilibrium in our founding mass-bred population. However, we cannot discount the possibility that some rare alleles were not captured or that some linkage disequilibrium persisted in our mass-bred population. The mass-bred population was maintained as one large panmictic population with initial numbers of approximately 1,800 individuals. During the 3 generations prior to the initiation of the experiment, the population was expanded and kept in 20 plastic bottles containing $50 \mathrm{~mL}$ of medium in 300-mL plastic bottles. Flies from each generation were mixed and then evenly divided across the 20 bottles. Density of flies was maintained at approximately 150 individuals per bottle, to ensure that population sizes were substantial prior to the onset of the experiment.

\section{Experimental Setup}

Nine experimental selection regimes were initiated related to temperature differences along the east coast of Australia. In 6 of these cabinets, temperatures mimicked patterns along the cline and followed the temperature model (see the appendix, available online), simulating natural variation within and among days. The temperatures of the selection regimes were on average $26.9^{\circ}, 23.9^{\circ}, 21.1^{\circ}, 18.9^{\circ}, 16.7^{\circ}$, and $13.4^{\circ} \mathrm{C}$. Three selection regimes with constant temperatures at $27^{\circ}, 22^{\circ}$, and $17^{\circ} \mathrm{C}$ were also initiated at the same time. Data loggers placed inside the bottles showed that humidity at each temperature was greater than $90 \%$ throughout a generation at each temperature regime. 
At the start of the experiment, flies from the large massbred population were collected and used as founders in the 9 selection regimes. For each selection regime, 4 replicate lines were initiated and independently maintained. Each line was maintained in 4 bottles, each containing approximately 200 adults (fig. A2; figs. A1, A2 are available online). Every generation, the 4 bottles per replicate were mixed to minimize drift and inbreeding within each replicate. Because of temperature-related fecundity differences across the selection regimes, flies were maintained in the bottles for different lengths of time, thereby ensuring that a similar number of flies eclosed from each bottle and selection regime. Each generation, ovipositing flies were transferred sequentially to fresh food 2 times, resulting in 2 oviposition periods involving flies of a different age. To avoid confounding effects of selection on early-life reproduction, which can result in correlated selection for a number of life-history traits (Rose and Charlesworth 1981; Khazaeli and Curtsinger 2013), flies for each generation were alternatively collected from the first or second egg-laying period. Furthermore, to avoid selection on development time, which could also contribute to correlated selection in other traits (Yadav and Sharma 2014), eclosing flies used to initiate the next generation were collected across a number of days (which varied depending on the temperature of the cabinet) until all flies had eclosed. Flies were collected every day without the use of $\mathrm{CO}_{2}$. These protocols were aimed at minimizing potential inadvertent selection for fast development time and other traits.

\section{Climate Model}

Selection regimes were set up to reflect summer/winter climate variation along the east coast of Australia. The extreme conditions were based on climate data from the cline ends, Tasmania and Cairns, over the past 10 years (www bom.gov.au). The most important differences in climatic variables were the changes in both mean and variance from north to south, with increasing variance and decreasing mean toward southern Australia (increasing latitude). To mimic these changes, we initiated a summer cline based on temperatures for the month of February from our cline ends, Tasmania and Cairns. Our initial objective was to run both summer and winter clines, but we found that the selection regimes simulating summer temperatures appropriately represented winter temperatures of another site along the latitudinal gradient - that is, winter temperatures for Cairns averaged $21.28^{\circ} \mathrm{C}$ with variance $10.66^{\circ} \mathrm{C}$, similar to a summer cabinet with mean $21.17^{\circ} \mathrm{C}$ and variance $13.10^{\circ} \mathrm{C}$. Thus, the different selection regimes reflected the "summer" cline, but winter conditions for a different location were also captured. However, to capture cooler conditions we also initiated one "Tasmanian spring" selection regime of $13.4^{\circ} \mathrm{C}$. The average temperature of this cabinet was based on the average temperatures of Tasmania in October. We did not use winter temperatures in Tasmania across June-August because these temperatures would have generated flies in a quiescent state and in reproductive diapause. Seasonal variation was not included in our model, as it was thought that this would reduce the selection intensity within a selection regime and limit our ability to detect changes over the short time frame represented by the experimental evolution experiment. A detailed description of the temperature model and an example of a 24-h temperature cycle generated through this mechanism is provided in the appendix. As we were concerned that extreme temperatures would result in the male sterility expected at a constant temperature around $31^{\circ} \mathrm{C}$ in Drosophila melanogaster (David et al. 2005), upper temperatures were bounded at $33^{\circ} \mathrm{C}$. Lower temperatures were less of an issue, with the minimum temperature of the coolest selection regime rarely falling below $7^{\circ} \mathrm{C}$. For the remaining selection regimes, we extrapolated the values on the basis of data from both ends of the gradient. Day length did not vary greatly between the cline ends $(1 \mathrm{~h})$, and therefore differences in light cycles across the cline were not included in the model $(12: 12 \mathrm{~h})$. Light intensities were varied during morning and night to reflect a dawn and dusk period, with light intensities slowly increasing and decreasing, respectively, over a 25 -min period.

\section{Viability and Density within the Selection Regimes}

To investigate selective pressures on the flies in the selection regimes, egg-to-adult viability (thought to be under strong temperature selection in the field; Mitrovski and Hoffmann 2001) was examined in the fluctuating selection regimes with mean temperatures of $26.9^{\circ}, 16.7^{\circ}$, and $13.4^{\circ} \mathrm{C}$ after 35,18 , and 8 generations of selection, respectively. Unlike in the ensuing experiments, where lines were removed from the selection regimes and reared at a constant temperature for 2 generations to control for environmental effects before testing, we here were interested in measuring egg-to-adult viability during the selection process. As a control, egg-to-adult viability was also measured in lines from the constant $22^{\circ} \mathrm{C}$ selection regime, when reared in the 3 fluctuating selection regimes with mean temperatures of $26.9^{\circ}, 16.7^{\circ}$, and $13.4^{\circ} \mathrm{C}$. To determine egg-to-adult viability, flies were placed onto spoons containing medium spread with live yeast and allowed to lay eggs for a $12-\mathrm{h}$ period. For each replicate line, 20 eggs were transferred into a vial, with 20 vials per replicate line, and placed into their respective selection regime. Emerging adults were then counted on eclosion.

Larval densities are known to influence body size and confound selection responses under different thermal re- 
gimes, influencing the detection of temperature-specific changes in traits (Santos et al. 1997; Santos et al. 2005). To test whether variation in density (and potential effects on size and productivity) influenced the observed results, density from the different selection regimes was assessed in a single generation by collecting and counting all flies emerging from a single laying period.

\section{Assessment of Clinal Patterns}

Prior to assessing traits, all lines from each cabinet were reared in a constant-temperature room set at $25^{\circ} \mathrm{C}$ for 2 generations. This allowed us to test for adaptive differences between populations without confounding environmental effects, including those due to cross-generational effects, such as maternal environmental effects. However, this design may not capture adaptive responses specific to the evolved environment. All traits were assessed in this manner after 1 year of selection. Cold and heat resistance were additionally assessed with this level of control in fluctuating selection regimes at 6 months, 1.5 years, and 3 years (table A2; tables A1-A8 are available online). For the final assessment after 3 years, only a subset of the selection regimes was assessed.

For all assessments, flies were controlled for density during development by ensuring that 40 eggs were added in each vial per line. On eclosion, flies were collected over a 48 -h period to reduce possible age effects. Both sexes were kept together for 2 days prior to separation to ensure that flies were mated. Sexes were separated with $\mathrm{CO}_{2}$ anesthesia followed by a minimum of $48 \mathrm{~h}$ of recovery prior to the assessment of traits. For all traits, only females were assayed. Because of the size of the experiment, traits were assessed using a block design, where an equal number of replicate individuals for each line were scored in each block. For all traits, we assayed between 14 and 20 individual females, although in a few cases fewer individuals were assessed (tables A3-A5). We assessed the stress traits heat knockdown, chill coma recovery, desiccation, and starvation resistance and the life-history traits development time, egg-to-adult viability, fecundity, and body size. For a detailed description, see the appendix.

\section{Analysis}

To examine the effects of selection regime on traits, we used a nested ANOVA run in SPSS (ver. 17), with replicate lines nested within selection regime. Trait assessments were performed in blocks, with replicates evenly distributed across the blocks and block included as a random effect in the final model. Selection regime was treated as a fixed factor, while replicate was treated as a random factor. For cold and heat resistance, the data did not meet the assump- tions of normality and homogeneity of variance and were consequently log transformed. All proportional viability data were transformed using arcsine-square root transformation, with confidence intervals (CIs) calculated via bootstrapping in SPSS. For all other traits, CIs were calculated by multiplying the SE by 1.96 . For full notation of all fitted models, see the appendix. We accounted for multiple comparison using the false-discovery-rate method (Verhoeven et al. 2005).

To examine directional patterns in trait responses across selection regimes, we constructed a nested generalized mixed model with average temperature as a covariate using the lme4 package (Bates et al. 2015) in R (R Development Core Team 2014). Selection regime was treated as a fixed factor, replicate nested within selection regime was treated as a random factor, and block was treated as a random factor. As we were interested in testing whether variance in temperature or alternative extreme conditions (as well as mean temperature) predicted trait evolution across the selection regimes, we included all possible predictor variables when testing the effects of the fluctuating regimes. Extreme conditions (number of hours above $30^{\circ}$ or below $14^{\circ} \mathrm{C}$ ) showed strong colinearity with temperature and were removed from the model. For all models, $R^{2}$ values were calculated for the marginal model (containing only fixed effects) and the conditional model (containing both fixed and random effects; Nakagawa and Holger 2013) using the MuMIn package in $\mathrm{R}$ (Barton 2015). To determine the significance of each term in the model, we used parametric bootstrapping and likelihood ratio tests to compare the full model with the null model as implemented in the pbkrtest package in R (Halekoh and Højsgaard 2014).

For cold, heat, and starvation resistance where we had temporal assessments of resistance, we also examined the role played by laboratory adaptation and generation number in shaping trait resistance across the selection regimes. As traits were compared across different generations, data were standardized by the mean and SD of each assessment. Generation data were analyzed using a generalized mixed model with both average temperature and generation number as covariates. Selection regime and generation time were deemed fixed factors, while replicate was nested within selection regime and treated as a random factor.

Finally, we analyzed temporal patterns for starvation, cold, and heat resistance within the selection regime that mimicked temperatures found at the site of the original source population (Coffs Harbour). In the absence of laboratory adaptation, we would predict no change in these traits because selection pressures should be comparable to those found in the natural population. Alternatively, if laboratory adaptation shapes resistance across all cabinets, we would predict directional changes in traits. We analyzed 
the data using a nested generalized linear mixed model, with generation as a covariate, selection regime as a fixed factor, and replicate line nested within selection regime as a random factor.

\section{Results}

\section{Selection Inside the Cabinets: Viability and Density}

To test whether selection was acting on immature stages across the temperature regimes, we examined egg-to-adult viability within three of the selection regimes (fluctuating temperature $26.9^{\circ}, 16.7^{\circ}$, and $13.4^{\circ} \mathrm{C}$ ), with the hypothesis that temperature selection would result in lower egg-toadult viability at the extremes of the temperature range (Mitrovski and Hoffmann 2001; Kristensen et al. 2015). As a control, we also examined egg-to-adult viability in lines derived and tested at a constant temperature of $22^{\circ} \mathrm{C}$. Egg-to-adult viability differed significantly across the selection regimes, with the highest viability of $86 \%$ (95\% CI: $83 \%-90 \%)$ in the $16.7^{\circ} \mathrm{C}$ selection regime (selection regime: $\mathrm{df}=3, F=7.16, P=.01$; replicate lines: $\mathrm{df}=8, F=12.80$, $P<.01)$. Using Dunnett's post hoc analysis, a significant decrease in egg-to-adult viability was detected between the control (constant $22^{\circ} \mathrm{C}$ ) and the two clinal extremes, $26.9^{\circ}$ and $13.4^{\circ} \mathrm{C}$, with viability decreases of $42 \%$ and $23 \%$, respectively.

To test the success of our attempts to reduce density variation across selection regimes, we collected and counted all adults from each selection regime over a single generation. We found a significant difference between selection regimes $\left(F_{5,18}=5.23, P<.01\right)$ driven by the fluctuating temperature selection regime with a mean temperature of $21.3^{\circ} \mathrm{C}$, which had the highest productivity of the selection regimes (average difference: $24.8 \%$ ). This was further confirmed by a Bonferroni post hoc analysis that demonstrated significant differences between the $21.3^{\circ} \mathrm{C}$ regime and the $26.9^{\circ}, 16.71^{\circ}$, and $13.42^{\circ} \mathrm{C}$ regimes (data not shown). This pattern might confound the detection of thermal responses (Santos et al. 1997), but because only the intermediate temperature treatment differed in density it should not affect our ability to detect linear temperature associations. In addition, removal of this selection regime from the generalized linear mixed models did not alter the results (data not shown).

\section{Assessment of Clinal Patterns}

Fluctuating Environments. All traits were assessed after 1 year of laboratory evolution (except fecundity, which was assessed after 1.5 years). Significant effects of selection regime involving the fluctuating conditions were detected for starvation resistance, development time, and body size (table 1). Developmental time and starvation resistance showed a significant linear relationship with average tem- perature across the selection regimes, such that lines that had evolved in the warmest selection regimes took longer to develop and were more starvation resistant (table 2; fig. 1), with an average change in development time of $2.5 \%$ and in starvation of $20 \%$ between the cline ends. For body size, temperature variance in the selection regimes was a better predictor of trait evolution than average temperature, with increased variance linked to a decrease in body size of around 1.62\% (table 2; fig. 2). Data underlying figures 1 and 2 are deposited in the Dryad Digital Repository: http://dx.doi.org/10.5061/dryad.t16c5 (Kellermann et al. 2015). While a significant selection effect for eggto-adult viability was detected, temperature variance was marginally nonsignificant $(P=.058)$ in the linear model. Although selection regime was not significant for cold resistance, there was a significant negative linear relationship between cold resistance and temperature variance; this relationship was counterintuitive, with lines in the warmest cabinet with the lowest temperature variance evolving the highest cold resistance. For all traits, there were significant replicate-line effects, reflecting divergence between lines in the cabinets (table 1).

Constant Environments. We found little evidence for selection regime shaping the evolution of traits in constant environments, with the exception of development time (tables 1,2). When measured in a common environment, development was quicker by an average of $3.12 \%$ in lines evolving in the cooler constant $17^{\circ} \mathrm{C}$ in comparison to the $22^{\circ}$ and $27^{\circ} \mathrm{C}$ regimes, which had the same development time. This result matched the direction of evolution in the fluctuating selection regimes.

\section{Temporal Assessments in Heat and Cold Resistance}

Heat and cold resistance were assessed 4 times over a 3-year period. Regardless of the generation of assessment, selection regime did not significantly alter thermal tolerances in the direction predicted by the temperature to which lines were exposed (table A7). Where a significant association between temperature and resistance was detected (cold resistance: assessment 1 for constant and assessment 4 for fluctuating conditions; heat resistance: assessment 1 for fluctuating conditions), responses were not in the predicted direction - that is, greater cold tolerance tended to occur after evolution in the warmest cabinet.

\section{Laboratory Adaptation}

For cold, heat, and starvation resistance, temporal assessments (3-4 times over a 3-year period) were used to investigate the possible role played by laboratory adaptation in shaping the evolution of traits inside the selection regimes. 
Table 1: ANOVA for all traits assessed after 1 year of laboratory natural selection

\begin{tabular}{|c|c|c|c|c|c|c|}
\hline \multirow[b]{2}{*}{ Trait } & \multicolumn{3}{|c|}{ Fluctuating selection regime } & \multicolumn{3}{|c|}{ Constant selection regime } \\
\hline & df & MS & $F$ & df & MS & $F$ \\
\hline \multicolumn{7}{|l|}{ Cold (log): } \\
\hline Selection regime & 5 & .045 & 1.363 & 2 & .045 & .970 \\
\hline Replicate (selection regime) & 18 & .033 & $1.730^{*}$ & 9 & .047 & $2.735^{* *}$ \\
\hline Block & 2 & .177 & $9.181^{* * * *}$ & 2 & .168 & $9.820^{* * *}$ \\
\hline Error & 420 & .019 & & 207 & .017 & \\
\hline \multicolumn{7}{|l|}{ Heat $(\log )$ : } \\
\hline Selection regime & 5 & .075 & 1.918 & 2 & .045 & .811 \\
\hline Replicate (selection regime) & 17 & .039 & $2.639^{* * * *}$ & 9 & .056 & $2.649^{* *}$ \\
\hline Block & 4 & .186 & $12.462^{* * * *}$ & 4 & .051 & $2.440^{*}$ \\
\hline Error & 385 & .015 & & 208 & .021 & \\
\hline \multicolumn{7}{|l|}{ Starvation: } \\
\hline Selection regime & 5 & $3,761.487$ & $3.842^{* *}$ & 2 & 428.720 & .443 \\
\hline Replicate (selection regime) & 18 & $1,018.998$ & $2.915^{* * *}$ & 8 & 964.056 & $3.840^{* * *}$ \\
\hline Error & 383 & 349.512 & & 187 & 251.072 & \\
\hline \multicolumn{7}{|l|}{ Development time: } \\
\hline Selection regime & 5 & 249.365 & $7.266^{* *}$ & 2 & $1,094.675$ & $26.640^{* *}$ \\
\hline Replicate (selection regime) & 15 & 34.644 & $2.711^{* *}$ & 6 & 41.974 & $3.587^{* *}$ \\
\hline Vial (replicate) & 57 & 9.893 & .774 & 36 & 13.569 & 1.159 \\
\hline Block & 1 & 44.818 & 3.507 & 1 & 17.451 & 1.491 \\
\hline Error & 381 & 12.780 & & 188 & 11.703 & \\
\hline \multicolumn{7}{|l|}{ Body size (variance): } \\
\hline Selection regime & 5 & .026 & $2.760^{* *}$ & 2 & .006 & .698 \\
\hline Replicate (selection regime) & 18 & .010 & $1.958^{*}$ & 9 & .009 & 1.597 \\
\hline Block & 1 & .304 & $58.473^{* * * *}$ & 1 & .243 & $44.808^{* * * *}$ \\
\hline Error & 750 & .005 & & 249 & .005 & \\
\hline \multicolumn{7}{|l|}{ Viability (variance): } \\
\hline Selection regime & 5 & 1.009 & $3.940^{*}$ & 2 & .109 & 1.701 \\
\hline Replicate (selection regime) & 15 & .59 & $8.446^{* * * *}$ & 6 & .064 & $2.345^{*}$ \\
\hline Vial (replicate) & 56 & .037 & 1.195 & 36 & .026 & .932 \\
\hline Error & 361 & .031 & & 182 & .027 & \\
\hline \multicolumn{7}{|l|}{ Desiccation: } \\
\hline Selection regime & 5 & 22.192 & .944 & 2 & 18.476 & .745 \\
\hline Replicate (selection regime) & 18 & 23.532 & $5.440^{* * * *}$ & 9 & 24.847 & $4.761^{* * *}$ \\
\hline Block & 4 & 7.401 & 1.711 & 4 & 13.739 & $2.632^{*}$ \\
\hline Error & 434 & 4.326 & & 218 & 5.219 & \\
\hline \multicolumn{7}{|l|}{ Fecundity: } \\
\hline Selection regime & 5 & 18.913 & .269 & $\ldots$ & $\ldots$ & $\ldots$ \\
\hline Replicate (selection regime) & 18 & 70.439 & 1.267 & $\ldots$ & $\ldots$ & $\cdots$ \\
\hline Error & 216 & 55.613 & & $\ldots$ & $\ldots$ & $\ldots$ \\
\hline
\end{tabular}

Note: Asterisks indicate $P$ values accounting for multiple comparisons using the false-discovery-rate method.

${ }^{*} P<.05$.

${ }^{* * *} P<.01$.

${ }^{* * *} P<.001$.

This was done in two ways. Initially, we examined the relationship between generation number and trait values. Under laboratory adaptation shaping trait evolution in a directional manner, a relationship between time in the laboratory (i.e., generation number) and trait mean might be expected. In contrast, an overriding relationship between temperature and trait means might indicate that tempera- ture selection rather than laboratory evolution drives trait evolution. We found no evidence for directional laboratory adaptation for cold, heat, and starvation resistance because there were no significant relationships between trait means and generation number (table 3 ).

Because laboratory adaptation may be masked by temperature selection, we also examined patterns of evolution 
Table 2: Summary of a generalized linear model examining the relationship between average temperature and trait evolution

\begin{tabular}{|c|c|c|c|c|c|c|}
\hline \multirow[b]{2}{*}{ Trait } & \multicolumn{3}{|c|}{ Fluctuating environments } & \multicolumn{3}{|c|}{ Constant environments } \\
\hline & Slope \pm SE & LRT & $R^{2}$ & Slope \pm SE & LRT & $R^{2}$ \\
\hline \multicolumn{7}{|c|}{ Chill coma recovery $(\log )$ : } \\
\hline Temperature & $-.005 \pm .002$ & 2.278 & $.022(.101)$ & $-.004 \pm .004$ & 1.233 & $.009(.186)$ \\
\hline Variance & $-.006 \pm .003$ & $8.227^{*}$ & & & & \\
\hline \multicolumn{7}{|c|}{ Heat knockdown (log): } \\
\hline Temperature & $.002 \pm .003$ & .202 & $.007(.271)$ & $.085 \pm .287$ & 2.862 & $.001(.129)$ \\
\hline Variance & $.003 \pm .004$ & .440 & & & & \\
\hline \multicolumn{7}{|l|}{ Starvation: } \\
\hline Temperature & $1.249 \pm .456$ & $10.321^{*}$ & $.074(.174)$ & $.154 \pm .550$ & -.098 & $.001(.133)$ \\
\hline Variance & $-.001 \pm .544$ & .001 & & & & \\
\hline \multicolumn{7}{|c|}{ Development time: } \\
\hline Temperature & $.361 \pm .084$ & $19.091^{*}$ & $.043(.077)$ & $.576 \pm .148$ & $10.835^{*}$ & $.070(.122)$ \\
\hline Variance & $.019 \pm .102$ & .039 & & & & \\
\hline \multicolumn{7}{|l|}{ Body size: } \\
\hline Temperature & $-.001 \pm .001$ & 1.235 & $.018(.172)$ & $.001 \pm .001$ & -.152 & $.001(.021)$ \\
\hline Variance & $-.003 \pm .001$ & $7.256^{*}$ & & & & \\
\hline \multicolumn{7}{|l|}{ Viability: } \\
\hline Temperature & $-.011 \pm .009$ & .022 & $.084(.371)$ & $-.005 \pm .004$ & .664 & $.008(.095)$ \\
\hline Variance & $-.027 \pm .011$ & 6.138 & & & & \\
\hline \multicolumn{7}{|l|}{ Desiccation: } \\
\hline Temperature & $.049 \pm .062$ & .956 & $.008(.200)$ & $.042 \pm .081$ & 2.562 & $.005(.192)$ \\
\hline Variance & $.003 \pm .075$ & .002 & & & & \\
\hline \multicolumn{7}{|l|}{ Fecundity: } \\
\hline Temperature & $.142 \pm .102$ & 2.552 & $.001(.005)$ & $\ldots$ & & . \\
\hline Variance & $.029 \pm .124$ & .066 & & & & \\
\hline
\end{tabular}

Note: The analysis is divided into lines evolving under fluctuating or constant selection regimes. The marginal $R^{2}$ value is given with the conditional $R^{2}$ value in parentheses, along with likelihood ratio test (LRT) values.

${ }^{*} P<.05$.

for cold, heat, and starvation resistance in the selection regime that mimicked the temperatures from which the original mass-bred population was collected (i.e., Coffs Harbour; average temperature of $21.1^{\circ} \mathrm{C}$ ). We expected that any changes in traits across generations under this selection regime would reflect laboratory adaptation rather than temperature selection because temperature selection should be negligible in this selection regime. We found no evidence for changes in traits across generations for either cold, heat, or starvation resistance in this environment (table 3). We therefore suspect that strong directional laboratory adaptation is unlikely to contribute to observed differences across selection regimes for cold, heat, and starvation resistance.

\section{Discussion}

After 1-3 years of laboratory natural selection, we found no evidence of a response to selection in heat and cold resistance, irrespective of whether selection was performed under constant or fluctuating thermal regimes. This result is in contrast to expectations based on results from studies of natural populations of Drosophila melanogaster collected at different latitudes (Hoffmann et al. 2002; Hoffmann and Weeks 2007; Sgro et al. 2010) and results from previous experimental evolution studies demonstrating temperature-driven divergence - albeit for only 3 constant temperatures - over a 4-year period (Huey et al. 1991; Cavicchi et al. 1995). Moreover, rapid responses in laboratory selection experiments (McColl et al. 1996; Anderson et al. 2005; Bubliy and Loeschcke 2005) highlight that heat and cold resistance are underpinned by significant variation that can readily be selected.

Temperature-driven responses were detected for starvation resistance, development time, and body size, with the establishment of significant laboratory clines within 1 year of laboratory evolution. The formation of these laboratory clines was not in the direction predicted from established trait clines in natural populations (James and Partridge 1995; James et al. 1995; Hoffmann et al. 2002, 2005), with the exception of development time. Similar to James et al. (1995), we found longer development times with increasing temperatures; however, these changes were small $(<3 \%$ between the cline ends). While flies from the warmest cab- 


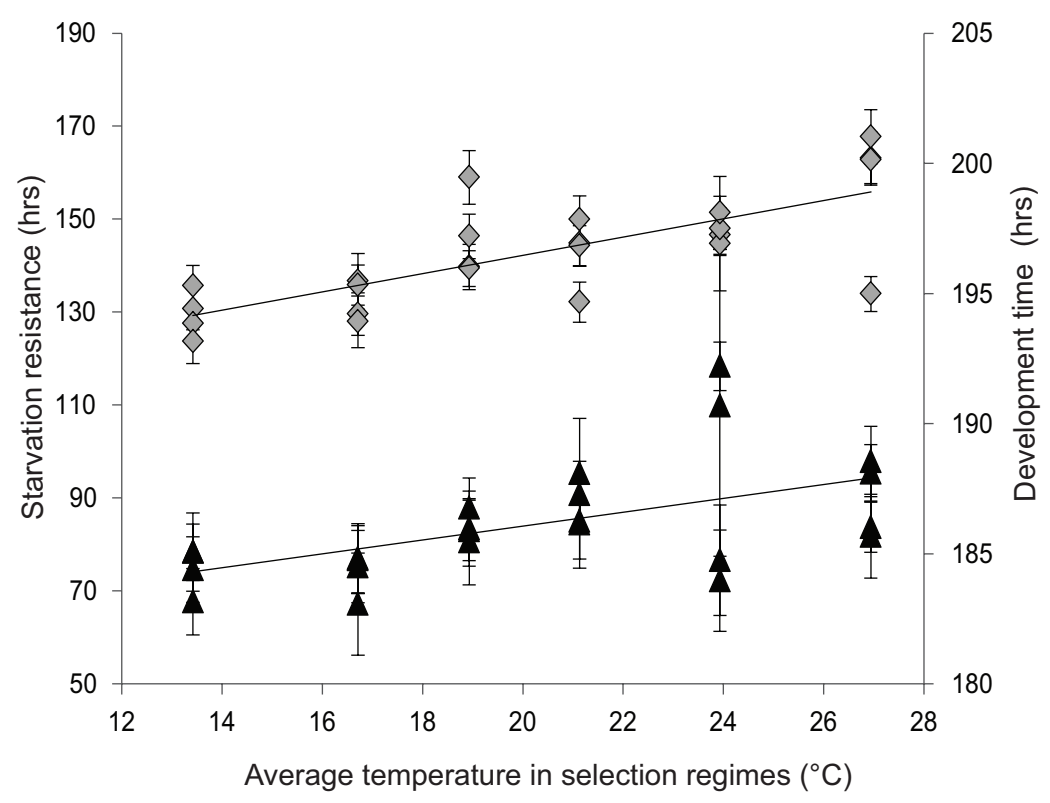

Figure 1: Relationship between development time (gray diamonds) and starvation resistance (black triangles) and average temperature across the fluctuating selection regimes after 1 year of laboratory adaptation. Error bars reflect $95 \%$ confidence intervals.

inet were $20 \%$ more starvation resistant than those from the coldest cabinet, this result contrasted with the absence of clinal patterns in Australia or South America and opposing clines on the Indian subcontinent (Karan et al. 1998; Robinson et al. 2000; Hoffmann et al. 2005; Parkash et al. 2012). Variation in temperature was more important than average temperature for predicting body size variation, but the link between temperature variation and latitudinal clines has not been explored. While latitudinal clines in body size have consistently been demonstrated

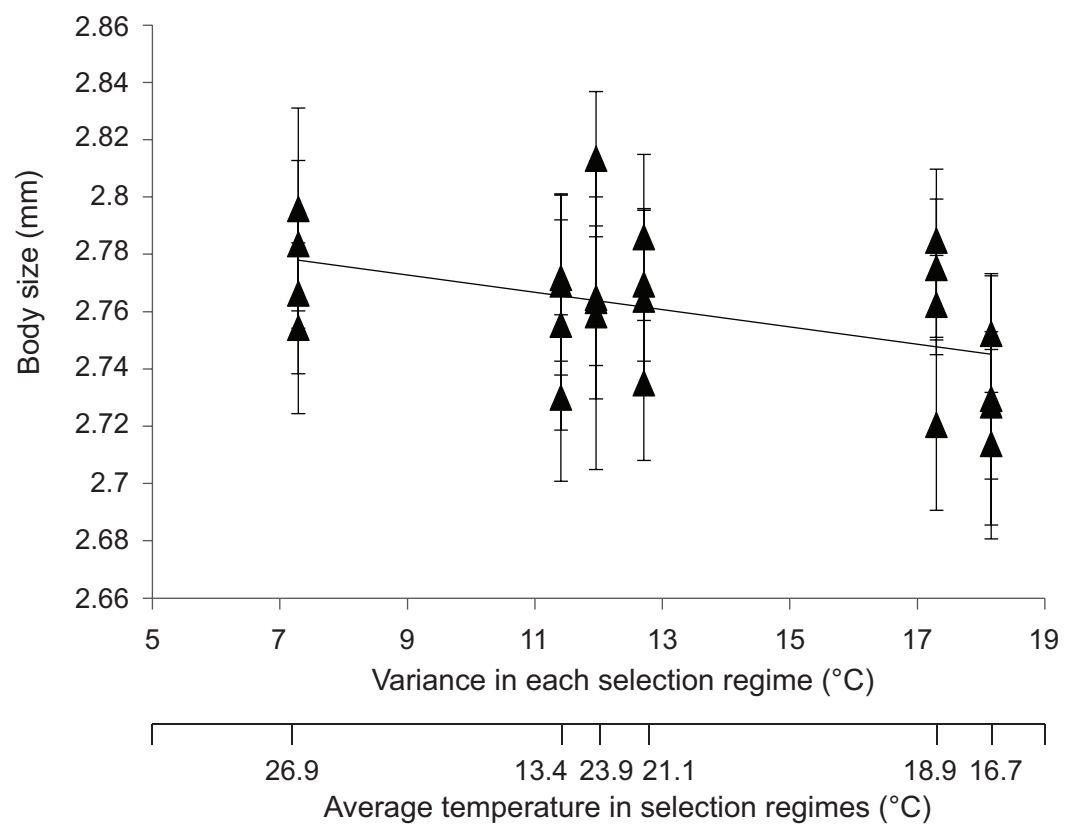

Figure 2: Relationship between body size and variance in temperature across the fluctuating selection regimes after 1 year of laboratory adaptation. The corresponding mean temperature of each selection regime is given as the secondary $X$-axis. Error bars reflect $95 \%$ confidence intervals. 
Table 3: Summary of results from generalized linear models examining the relationship between generation and heat, cold, and starvation resistance

\begin{tabular}{|c|c|c|c|}
\hline Trait & Slope \pm SE & LRT & $R^{2}$ \\
\hline \multicolumn{4}{|c|}{ Fluctuating selection regimes: } \\
\hline \multicolumn{4}{|c|}{ Chill coma recovery: } \\
\hline Temperature & $-.009 \pm .007$ & 3.849 & \multirow[t]{2}{*}{$.004(.007)$} \\
\hline Generation & $-.002 \pm .002$ & .778 & \\
\hline \multicolumn{4}{|c|}{ Heat knockdown: } \\
\hline Temperature & $-.003 \pm .024$ & .504 & \multirow[t]{2}{*}{$.001(.010)$} \\
\hline Generation & $-.006 \pm .007$ & 1.015 & \\
\hline \multicolumn{4}{|l|}{ Starvation: } \\
\hline Temperature & $.042 \pm .014$ & $5.406^{*}$ & \multirow[t]{2}{*}{$.023(.079)$} \\
\hline Generation & $-.005 \pm .002$ & 4.283 & \\
\hline \multicolumn{4}{|c|}{ Coffs Harbour selection regime: } \\
\hline \multicolumn{4}{|c|}{ Chill coma recovery: } \\
\hline Generation & $-.003 \pm .004$ & .432 & $.003(.034)$ \\
\hline \multicolumn{4}{|c|}{ Heat knockdown: } \\
\hline Generation & $-.005 \pm .003$ & 1.342 & $.007(.033)$ \\
\hline \multicolumn{4}{|l|}{ Starvation: } \\
\hline Generation & $-.015 \pm .004$ & 4.436 & $.058(.156)$ \\
\hline
\end{tabular}

Note: The analysis is split into lines from fluctuating selection regimes and a single selection regime that mimics temperatures similar to the original source population from Coffs Harbour. The marginal $R^{2}$ value is given with the conditional $R^{2}$ value in parentheses, along with likelihood ratio test (LRT) values.

${ }^{*} P<.05$.

in Drosophila, in line with Bergmann's rule with a decrease in size toward the tropics (James et al. 1995; Imasheva et al. 2000; Hallas et al. 2002), we found that flies from the warmest cabinet eclosed at a larger body size, although the size difference between cline ends was small ( $<3 \%)$. Past experiments have found that experimental evolution at higher temperatures decreases size (Cavicchi et al. 1985), but there is a potential confounding effect of density in interpretations of such experiments (Santos et al. 2005), which were carefully minimized in the current experiment. Unlike cold resistance, heat resistance, desiccation resistance, and, to a lesser extent, viability, which have been closely linked to environmental variables (Mitrovski and Hoffmann 2001; Kellermann et al. 2012a, 2012b), the main drivers of variation in starvation resistance, developmental time, and body size are not as clear (Parkash and Munjal 2000).

The mismatch between the results presented here and those from natural populations suggests that trait evolution in natural populations depends on factors other than temperature. One possible explanation is that pleiotropic interactions among alleles influence the evolution of traits and that these interactions are different in a laboratory environment than in the wild (Hoffmann and Harshman 1999; Kawecki et al. 2012). Under laboratory conditions, where resources are readily available, alleles that are deleterious in natural populations may be neutral. As a consequence, evolution in the laboratory may be fundamentally different from evolution in natural populations. Differences in genetic background and experimental methods may also contribute to the contrasting results of the current study and those of Huey et al. (1991) and Cavicchi et al. (1995), who found temperature-driven divergence in heat and cold resistance. In the current study, care was taken to remove confounding effects of selection on life-history traits, development time, and early-life reproduction, resulting in a methodology different from that used by Huey et al. (1991) and Cavicchi et al. (1995).

Experimental evolution studies have design limitations, particularly when working with a species where measurements cannot be directly related back to those in the ancestral population. In particular, laboratory adaptation may confound evolutionary responses (Simoes et al. 2008), with good evidence for trait changes due to laboratory adaptation in Drosophila species (Hoffmann et al. 2001; Simoes et al. 2008). We attempted to test for directional evolutionary changes like those detected by Griffiths et al. (2005) by monitoring temporal changes in starvation, cold, and heat resistance. The absence of such changes suggests that directional laboratory adaptation is unlikely to explain our results. It is possible that laboratory adaptation was nondirectional, driven by opposing effects of selection and laboratory adaptation. We examined temporal changes in traits in a single selection regime mimicking temperatures of the original source population, where we predicted that directional changes would be driven by laboratory adapta- 
tion rather than selection, and we found no evidence for laboratory adaptation influencing the observed results.

Selection intensities in our treatments may have been weak; however, we did find lower egg-to-adult viability under the more extreme conditions, suggesting the potential for selection on the traits studied, and clearly some traits did respond to selection. Experimental temperatures may not reflect relevant extremes compared with natural populations, with extreme events/temperatures linked to species distributions (Overgaard et al. 2014). This is complicated by our limited understanding of temperatures experienced in natural populations and the extent to which species behaviorally thermoregulate (Kellermann et al. 2012b; Sunday et al. 2014). Finally, genetic drift may also reduce selection responses and will be most pervasive when population sizes are small and selection intensities weak. Significant divergence between replicate lines as detected in our study may be indicative of drift, although this did not prevent some selection responses being detectable. Line divergence is a general feature of selection responses in Drosophila regardless of attempts to ensure that the same selection pressures are applied (Cohan and Hoffmann 1986).

Experimental evolution studies have influenced our understanding of evolutionary theory (for a review, see Kawecki et al. 2012) and are increasingly being used to examine responses to stressful temperatures (Collins and Bell 2004; Munday et al. 2013; Reusch and Boyd 2013; Schou et al. 2014). Evolutionary responses are commonly observed (Kassen 2002; Kawecki et al. 2012; Lohbeck et al. 2012); however, examples of limited or unexpected responses to selection have also emerged (Collins and Bell 2004; Schou et al. 2014). The difficulty with experimental evolution studies is quantifying whether the observed null results - that is, a lack of response - is reflective of a true evolutionary constraint/limitation or simply a by-product of laboratory experimental designs. Here we found that the results from laboratory experiments were not good at predicting evolutionary responses in natural populations. The mismatch between laboratory evolution and natural trait variation highlights the caution that needs to be taken when inferring evolutionary processes in laboratory environments and builds on previous studies that show a lack of congruity between performance of selection lines under laboratory and field conditions (Kristensen et al. 2007). Moving from laboratory-based experimental evolution studies to field-based experimental evolution may provide an additional route to predicting evolutionary responses in natural populations.

\section{Acknowledgments}

We are grateful to N.-S. Bøgh for help with the temperature model; to D. Andersen, G. Azubi, L. Ohn-Andersen,
P. Sarup, M. F. Schou, L. van Koll, and J. Wit for technical assistance; to the Danish Natural Science Research Council (FNU) for financial support to V.L. (frame grant) and T.N.K. (Sapere aude stipend DFF-4002-00036); and to the Australian Research Council for financial support to V.K. and A.A.H. through its Discovery and Fellowship schemes.

\section{Literature Cited}

Anderson, A. R., A. A. Hoffmann, and S. W. McKechnie. 2005. Response to selection for rapid chill-coma recovery in Drosophila melanogaster: physiology and life-history traits. Genetical Research 85:15-22.

Barrett, R. D. H., A. Paccard, T. M. Healy, S. Bergek, P. M. Schulte, D. Schluter, and S. M. Rogers. 2011. Rapid evolution of cold tolerance in stickleback. Proceedings of the Roval Society B: Biological Sciences 278:233-238.

Barton, K. 2015. MuMIn: multi-model inference. http://CRAN.R -project.org/package $=$ MuMIn

Bates, D., M. Maechler, B. Bolker, and S. Walker. 2015. lme4: linear mixed effects models using Eigen and S4. R package version 1.1-8. http://CRAN.R-project.org/package $=$ lme 4 .

Bubliy, O. A., and V. Loeschcke. 2005. Correlated responses to selection for stress resistance and longevity in a laboratory population of Drosophila melanogaster. Journal of Evolutionary Biology 18:789803.

Cavicchi, S., D. Guerra, G. Giorgi, and C. Pezzoli. 1985. Temperature related divergence in experimental populations of Drosophila melanogaster. I. Genetic and developmental basis of wing size and shape variation. Genetics 109:665-689.

Cavicchi, S., D. Guerra, V. Latorre, and R. B. Huey. 1995. Chromosomal analysis of heat shock tolerance in Drosophila melanogaster evolving at different temperatures in the laboratory. Evolution 49:676-684.

Chevin, L. M., R. Lande, and G. M. Mace. 2010. Adaptation, plasticity, and extinction in a changing environment: towards a predictive theory. PLoS Biology 8:e1000357.

Cohan, F. M., and A. A. Hoffmann. 1986. Genetic divergence under uniform selection. II. Different responses to selection for knockdown resistance to ethanol among Drosophila melanogaster populations and their replicate lines. Genetics 114:145-164.

Collins, S. 2011. Competition limits adaptation and productivity in a photosynthetic alga at elevated $\mathrm{CO}_{2}$. Proceedings of the Roval Society B: Biological Sciences 278:247-255.

Collins, S., and G. Bell. 2004. Phenotypic consequences of 1,000 generations of selection at elevated $\mathrm{CO}_{2}$ in a green alga. Nature 431:566-569.

David, J. R., L. O. Araripe, M. Chakir, H. Legout, B. Lemos, G. Petavy, C. Rohmer, et al. 2005. Male sterility at extreme temperatures: a significant but neglected phenomenon for understanding Drosophila climatic adaptations. Journal of Evolutionary Biology 18:838-846.

Griffiths, J. A., M. Schiffer, and A. A. Hoffmann. 2005. Clinal variation and laboratory adaptation in the rainforest species Drosophila birchii for stress resistance, wing size, wing shape and development time. Journal of Evolutionary Biology 18:213-222.

Halekoh, U., and S. Højsgaard. 2014. A Kenward-Roger approximation and parametric bootstrap methods for tests in linear mixed models: the $\mathrm{R}$ package pbkrtest. Journal of Statistical Software 59:1-30. 
Hallas, R., M. Schiffer, and A. A. Hoffmann. 2002. Clinal variation in Drosophila serrata for stress resistance and body size. Genetical Research 79:141-148.

Hargreaves, A. L., K. E. Samis, and C. G. Eckert. 2014. Are species' range limits simply niche limits writ large? a review of transplant experiments beyond the range. American Naturalist 183:157-173.

Hoffmann, A. A., A. Anderson, and R. Hallas. 2002. Opposing clines for high and low temperature resistance in Drosophila melanogaster. Ecology Letters 5:614-618.

Hoffmann, A. A., R. Hallas, C. Sinclair, and L. Partridge. 2001. Rapid loss of stress resistance in Drosophila melanogaster under adaptation to laboratory culture. Evolution 55:436-438.

Hoffmann, A. A., and L. G. Harshman. 1999. Desiccation and starvation resistance in Drosophila: patterns of variation at the species, population and intrapopulation levels. Heredity 83:637-643.

Hoffmann, A. A., and C. M. Sgro. 2011. Climate change and evolutionary adaptation. Nature 470:479-485.

Hoffmann, A. A., J. Shirriffs, and M. Scott. 2005. Relative importance of plastic vs genetic factors in adaptive differentiation: geographical variation for stress resistance in Drosophila melanogaster from eastern Australia. Functional Ecology 19:222-227.

Hoffmann, A. A., J. G. Sorensen, and V. Loeschcke. 2003. Adaptation of Drosophila to temperature extremes: bringing together quantitative and molecular approaches. Iournal of Thermal Biology 28:175-216.

Hoffmann, A. A., and A. R. Weeks. 2007. Climatic selection on genes and traits after a 100 year-old invasion: a critical look at the temperate-tropical clines in Drosophila melanogaster from eastern Australia. Genetica 129:133-147.

Huey, R. B., L. Partridge, and K. Fowler. 1991. Thermal sensitivity of Drosophila melanogaster responds rapidly to laboratory natural selection. Evolution 45:751-756.

Imasheva, A. G., B. Moreteau, and J. R. David. 2000. Growth temperature and genetic variability of wing dimensions in Drosophila: opposite trends in two sibling species. Genetical Research 76: 237-247.

James, A. C., R. B. R. Azevedo, and L. Partridge. 1995. Cellular basis and developmental timing in a size cline of Drosophila melanogaster. Genetics 140:659-666.

James, A. C., and L. Partridge. 1995. Thermal evolution of rate of larval development in Drosophila melanogaster in laboratory and field populations. Journal of Evolutionary Biology 8:315-330.

Karan, D., N. Dahiya, A. K. Munjal, P. Gibert, B. Moreteau, R. Parkash, and J. R. David. 1998. Desiccation and starvation tolerance of adult Drosophila: opposite latitudinal clines in natural populations of three different species. Evolution 52:825-831.

Kassen, R. 2002. The experimental evolution of specialists, generalists, and the maintenance of diversity. Journal of Evolutionary Biology 15:173-190.

Kawecki, T. J., R. E. Lenski, D. Ebert, B. Hollis, I. Olivieri, and M. C. Whitlock. 2012. Experimental evolution. Trends in Ecology and Evolution 27:547-560

Kellermann, V., A. A. Hoffmann, T. N. Kristensen, N. N. Moghadam, and V. Loeschcke. 2015. Data from: Experimental evolution under fluctuating thermal conditions does not reproduce patterns of adaptive clinal differentiation in Drosophila melanogaster. American Naturalist, Dryad Digital Repository, http:// dx.doi.org/10.5061/dryad.t16c5.

Kellermann, V., V. Loeschcke, A. A. Hoffmann, T. N. Kristensen, C. Flojgaard, J. R. David, J.-C. Svenning, et al. 2012a. Phylogenetic constraints in key functional traits behind species' climate niches: patterns of desiccation and cold resistance across 95 Drosophila species. Evolution 66:3377-3389.

Kellermann, V., J. Overgaard, A. A. Hoffmann, C. Flojgaard, J.-C. Svenning, and V. Loeschcke. 2012b. Upper thermal limits of Drosophila are linked to species distributions and strongly constrained phylogenetically. Proceedings of the National Academv of Sciences of the USA 109:16228-16233.

Khazaeli, A. A., and J. W. Curtsinger. 2013. Pleiotropy and life history evolution in Drosophila melanogaster: uncoupling life span and early fecundity. Lournals of Gerontology Series A: Biological Sciences and Medical Sciences 68:546-553.

Kristensen, T. N., V. Loeschcke, and A. A. Hoffmann. 2007. Can artificially selected phenotypes influence a component of field fitness? thermal selection and fly performance under thermal extremes. Proceedings of the Royal Society B: Biological Sciences 274:771-778.

Kristensen, T. N., J. Overgaard, J. Lassen, A. A. Hoffmann, and C. Sgro. 2015. Low evolutionary potential for egg-to-adult viability in Drosophila melanogaster at high temperatures. Evolution 69: 803-814.

Lohbeck, K. T., U. Riebesell, and T. B. H. Reusch. 2012. Adaptive evolution of a key phytoplankton species to ocean acidification. Nature Geoscience 5:346-351.

McColl, G., A. A. Hoffmann, and S. W. McKechnie. 1996. Response of two heat shock genes to selection for knockdown heat resistance in Drosophila melanogaster. Genetics 143:1615-1627.

Merila, J., and A. P. Hendry. 2014. Climate change, adaptation, and phenotypic plasticity: the problem and the evidence. Evolutionary Applications 7:1-14.

Mitrovski, P., and A. A. Hoffmann. 2001. Postponed reproduction as an adaptation to winter conditions in Drosophila melanogaster: evidence for clinal variation under semi-natural conditions. Proceedings of the Roval Society B: Biological Sciences 268:2163-2168.

Munday, P. L., R. R. Warner, K. Monro, J. M. Pandolfi, and D. J. Marshall. 2013. Predicting evolutionary responses to climate change in the sea. Ecology Letters 16:1488-1500.

Nakagawa, S., and S. Holger. 2013. A general and simple method for obtaining $R^{2}$ from generalized linear mixed-effects models. Methods in Ecology and Evolution 4:133-142.

Overgaard, J., M. R. Kearney, and A. A. Hoffmann. 2014. Sensitivity to thermal extremes in Australian Drosophila implies similar impacts of climate change on the distribution of widespread and tropical species. Global Change Biology 20:1738-1750.

Overgaard, J., T. N. Kristensen, and J. G. Sørensen. 2012. Validity of thermal ramping assays used to assess thermal tolerance in arthropods. PLoS ONE 7:e32758.

Parkash, R., D. D. Aggarwal, and B. Kalra. 2012. Coadapted changes in energy metabolites and body color phenotypes for resistance to starvation and desiccation in latitudinal populations of $D$. melanogaster. Evolutionary Ecology 26:149-169.

Parkash, R., and A. K. Munjal. 2000. Evidence of independent climatic selection for desiccation and starvation tolerance in Indian tropical populations of Drosophila melanogaster. Evolutionary Ecology Research 2:685-699.

R Development Core Team. 2014. R: a language and environment for statistical computing. R Foundation for Statistical Computing, Vienna. http://www.R-project.org/.

Reusch, T. B. H., and P. W. Boyd. 2013. Experimental evolution meets marine phytoplankton. Evolution 67:1849-1859. 
Rezende, E. L., M. Tejedo, and M. Santos. 2011. Estimating the adaptive potential of critical thermal limits: methodological problems and evolutionary implications. Functional Ecology 25:111121.

Robinson, S. J. W., B. Zwaan, and L. Partridge. 2000. Starvation resistance and adult body composition in a latitudinal cline of Drosophila melanogaster. Evolution 54:1819-1824.

Rose, M. R., and B. Charlesworth. 1981. Genetics of life-history in Drosophila melanogaster. II. Exploratory selection experiments. Genetics 97:187-196.

Santos, M., D. J. Borash, A. Joshi, N. Bounlutay, and L. D. Mueller. 1997. Density-dependent natural selection in Drosophila: evolution of growth rate and body size. Evolution 51:420-432.

Santos, M., W. Céspedes, J. Balanyà, V. Trotta, F. C. F. Calboli, A. Fontdevila, and L. Serra. 2005. Temperature-related genetic changes in laboratory populations of Drosophila subobscura: evidence against simple climatic-based explanations for latitudinal clines. American Naturalist 165:258-273.

Scheiner, S. M. 2002. Selection experiments and the study of phenotypic plasticity. Journal of Evolutionary Biology 15:889-898.

Schou, M. F., T. N. Kristensen, V. Kellermann, C. Schlotterer, and V. Loeschcke. 2014. A Drosophila laboratory evolution experiment points to low evolutionary potential under increased temperatures likely to be experienced in the future. Journal of Evolutionary Biology 27:1859-1868.

Sgro, C. M., J. Overgaard, T. N. Kristensen, K. A. Mitchell, F. E. Cockerell, and A. A. Hoffmann. 2010. A comprehensive assess- ment of geographic variation in heat tolerance and hardening capacity in populations of Drosophila melanogaster from eastern Australia. Journal of Evolutionary Biology 23:2484-2493.

Simoes, P., J. Santos, I. Fragata, L. D. Mueller, M. R. Rose, and M. Matos. 2008. How repeatable is adaptive evolution? the role of geographical origin and founder effects in laboratory adaptation. Evolution 62:1817-1829.

Sunday, J. M., A. E. Bates, M. R. Kearney, R. K. Colwell, N. K. Dulvy, J. T. Longino, and R. B. Huey. 2014. Thermal-safety margins and the necessity of thermoregulatory behavior across latitude and elevation. Proceedings of the National Academv of Sciences of the USA 111:5610-5615.

van Heerwaarden, B., and C. M. Sgro. 2011. The effect of developmental temperature on the genetic architecture underlying size and thermal clines in Drosophila melanogaster and D. simulans from the east coast of Australia. Evolution 65:1048-1067.

Verhoeven, K. J. F., K. L. Simonsen, and L. M. McIntyre. 2005. Implementing false discovery rate control: increasing your power. Oikos 108:643-647.

Yadav, P., and V. K. Sharma. 2014. Correlated changes in life history traits in response to selection for faster pre-adult development in

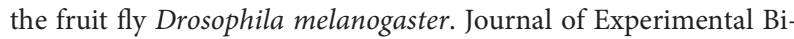
ology 217:580-589.

Associate Editor: Ian Dworkin Editor: Troy Day
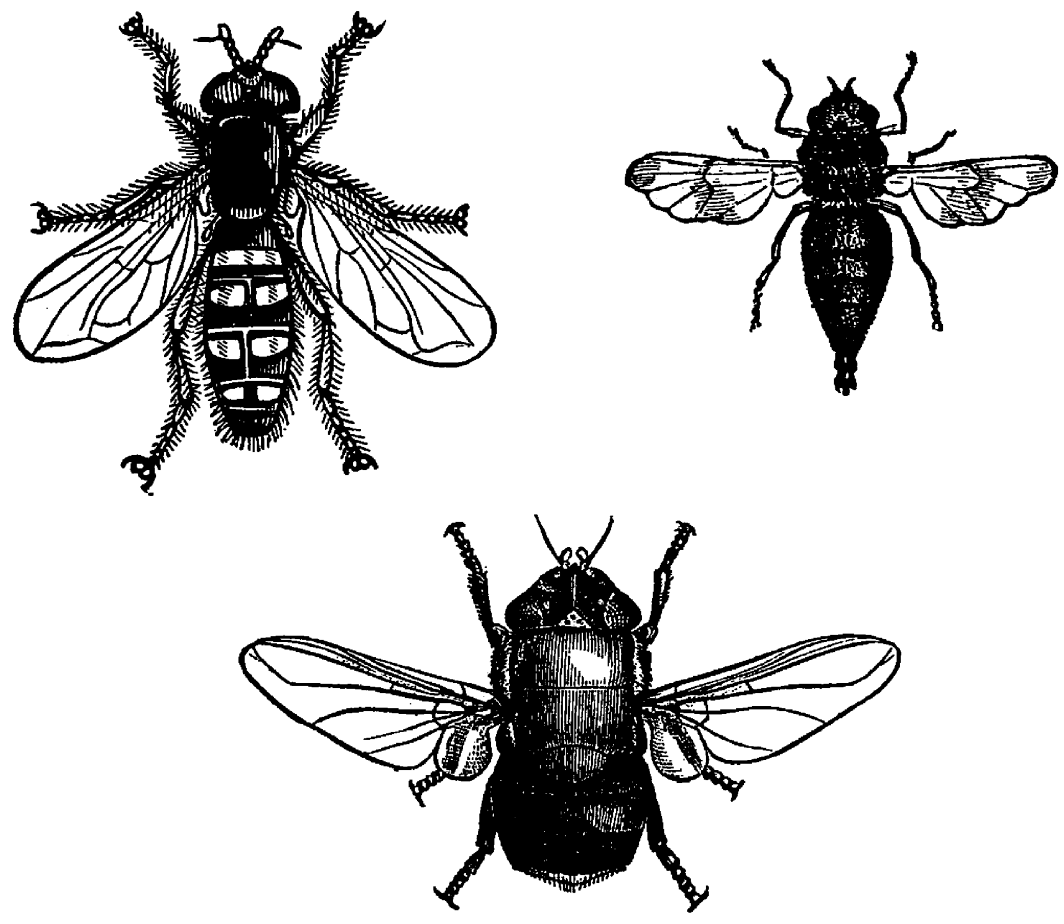

"The subject of flies becomes of vast moment to a Pharaoh, whose ears are dinned with the buzz of myriad winged plagues, mingled with angry cries from malcontent and fly-pestered subjects; or to the summer traveller in northern lands, where they oppose a stronger barrier to his explorations than the loftiest mountains or the broadest streams." From "A Chapter on Flies" by A. S. Packard Jr. (The American Naturalist, 1869, 2:586-596). 


\section{University Library}

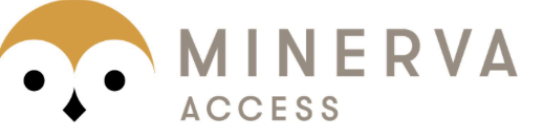

A gateway to Melbourne's research publications

Minerva Access is the Institutional Repository of The University of Melbourne

Author/s:

Kellermann, V;Hoffmann, AA;Kristensen, TN;Moghadam, NN;Loeschcke, V

Title:

Experimental Evolution under Fluctuating Thermal Conditions Does Not Reproduce Patterns of Adaptive Clinal Differentiation in Drosophila melanogaster

Date:

2015-11-01

Citation:

Kellermann, V., Hoffmann, A. A., Kristensen, T. N., Moghadam, N. N. \& Loeschcke, V. (2015). Experimental Evolution under Fluctuating Thermal Conditions Does Not Reproduce Patterns of Adaptive Clinal Differentiation in Drosophila melanogaster. AMERICAN NATURALIST, 186 (5), pp.582-593. https://doi.org/10.1086/683252.

Persistent Link:

http://hdl.handle.net/11343/115374 